\title{
Hybrid Algorithmic Approach for Medical Image Compression Based on Discrete Wavelet Transform (DWT) and Huffman Techniques for Cloud Computing
}

\author{
Prof D. Ravichandran, Ashwin Dhivakar M R and Dr. Vijay Dakha
}

\begin{abstract}
As medical imaging facilities move towards complete filmless imaging and also generate a large volume of image data through various advance medical modalities, the ability to store, share and transfer images on a cloud-based system is essential for maximizing efficiencies. The major issue that arises in teleradiology is the difficulty of transmitting large volume of medical data with relatively low bandwidth. Image compression techniques have increased the viability by reducing the bandwidth requirement and cost-effective delivery of medical images for primary diagnosis. Wavelet transformation is widely used in the fields of image compression because they allow analysis of images at various levels of resolution and good characteristics. The algorithm what is discussed in this paper employs wavelet toolbox of MATLAB. Multilevel decomposition of the original image is performed by using Haar wavelet transform and then image is quantified and coded based on Huffman technique. The wavelet packet has been applied for reconstruction of the compressed image. The simulation results show that the algorithm has excellent effects in the image reconstruction and better compression ratio and also study shows that valuable in medical image compression on cloud platform.
\end{abstract}

Keywords-Wavelet Packet, Discrete Wavelet Transform (DWT), Lossless image compression, Medical image, Huffman coding, Cloud computing

\section{Introduction}

In recent years, many studies have been made on wavelets. An excellent overview of what wavelets have brought to the fields as diverse as biomedical applications, wireless communications, computer graphics or turbulence, is given in [1].

Prof. D Ravichandran, Dept of Computer Science and Engineering Aurora Technological and Research Institute (ATRI)

Hyderabad, India

Ashwin Dhivakar M R, Dept of Computer and System Sciences Jaipur National University

India

Dr. Vijay Dhaka, Professor and Head of the Department

Computer Science and Engineering

Jaipur National University

India
Image compression is one of the most visible applications of wavelets [2]. It is well known that medical imaging facilities move towards complete filmless imaging and that compression plays a vital role for storing, transferring and sharing the patient's images across the computer system for primary diagnostics [3]-[6].

Compression is the process of reducing large data files into smaller files for efficiency of storage and transmission. In general, image compression techniques can be classified into two types, namely, lossy and lossless. In lossless image compression, the reconstructed image from the compressed data is identical to the original image. In lossy compression, the reconstructed image from the compressed data is not the same as that of original image. They are various techniques and methods employed for image compression in both lossy and lossless. Although lossy compression techniques yield high compression ratios, the medical community has been reluctant to adopt these techniques due to legal risks and they prefer to use lossless compression techniques despite low compression rates. The aim and goal of the compression algorithm is to maximize the compression ratio and minimize the mean square error of the image [7]-[9].

This paper presents comprehensive study with performance analysis of medical image compression based on Discrete Wavelet Transform (DWT) and Huffman techniques. Motivation to this work was seeking new methods of quantitative analysis of lossless image compression for medical images on cloud platform [3]. Wavelets appear to be a suitable tool for this task, because they allow analysis of images at various levels of resolution. Compression is achieved by the removal of redundant data. JPEG-2000 is a recently developed compression standard for still images that is based on Discrete Wavelet Transform (DWT) technique [10]. DWT image compression includes decomposition (transform of image), detail coefficients thresholding, and entropy encoding (Fig 1- a,b).

(a) Coding Section

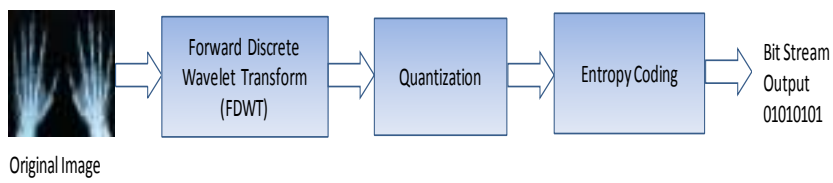


(b) Decoding Section

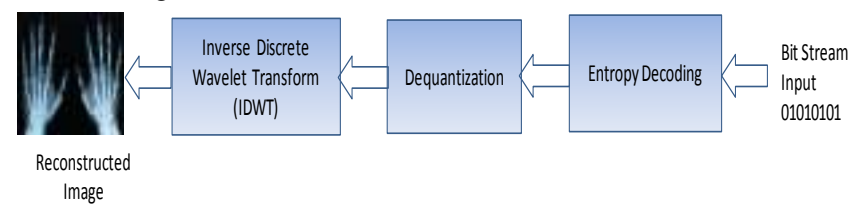

Figure 1 The process of Image Compression based on Wavelet Transform

This paper is organized as follows. Section 2 discusses the fundamentals of Discrete Wavelet Transform (DWT). Section 3 talks about the proposed work and research methodology of the hybrid algorithm. Section 4 cites the performance and analysis of the implementation steps of the proposed algorithm. Section 5 gives the conclusion and future work

\section{Discrete Wavelets Transform}

Wavelets are functions defined over a finite interval and have an average value of zero. The basic idea of the wavelet transform is to represent any arbitrary function ( $t$ ) as a superposition of a set of such wavelets or basis functions.
An image is a two Dimensional signal (2D) and that is considered to be matrices with $\mathrm{N}$ rows and $\mathrm{M}$ columns. In wavelet transform, decomposition of an image consists of two parts, one is lower frequency or approximation of an image (scaling function) and another is higher frequency or detailed part of an image (wavelet function). At every level of decomposition, the four sub-images are obtained, the approximation (LL), the vertical detail (LH), the horizontal detail (HL), and the diagonal detail (HH).

Then all the coefficients are discarded, except the LL coefficients that are transformed into the second level (Fig 2). To achieve the required compression ratio the LL coefficients are passed through a constant scaling factor [13].

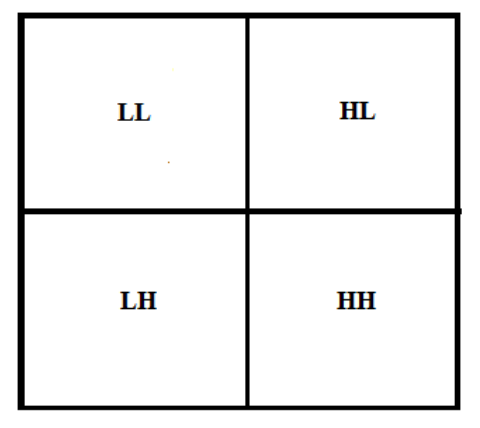

Ist $^{\text {st }}$ evel Decomposition

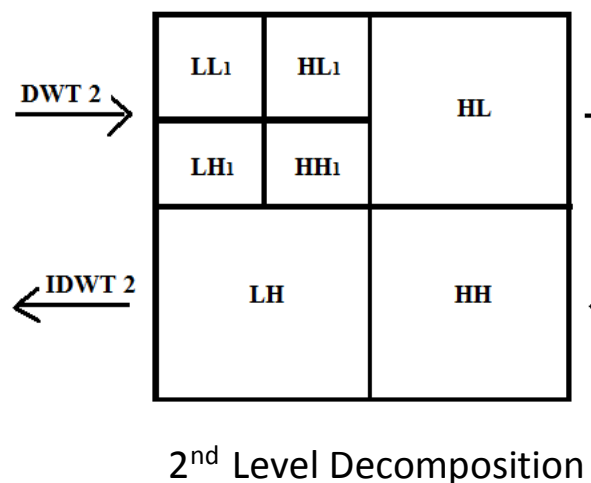

$2^{\text {nd }}$ Level Decomposition

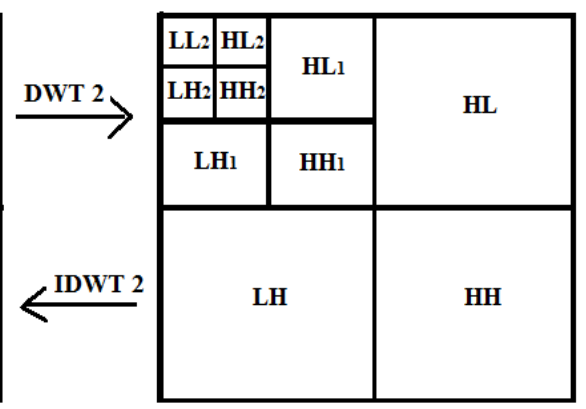

$3^{\text {rd }}$ Level Decomposition

Figure $2 \mathrm{Sub}$ band notations in the hierarchical Wavelet Transform

These basis functions or baby wavelets are obtained from a single prototype wavelet called the mother wavelet, by dilations or contractions (scaling) and translations (shifts). The main feature of DWT is multiscale representation of function.

By using the wavelets, given function can be analyzed at various levels of resolution. The DWT is also invertible and can be orthogonal [11]. Wavelet transform has emerged as very powerful tool for data compression [12].
After applying forward discrete wavelet transform, one can employ proper method to process the image coefficients for achieving effective compression [15]. For reconstructing the cosefficients are rescaled followed by padding zeros and then passed through wavelet filter. 


\section{Proposed Work And Environment Used}

The research methodology of the proposed work and algorithm steps are summarized as follows(Fig 3):

1. Load Image in MATLAB using Image Acquisition

2. Covert RGB to Gray scale and draw histogram of the original image.

3. Apply multilevel decomposition using 2D- DWT based on HAAR mother wavelet.

4. Apply histogram probability reduction function on RGB components using Mean intensities.

5. Apply Image quantization

6. Calculate probability index for each unique quantity.

7. Calculate unique binary code of Huffman code for each unique symbol.

8. Apply Huffman compression using Huffman tree.

9. Calculate CR, MSE and PSNR.

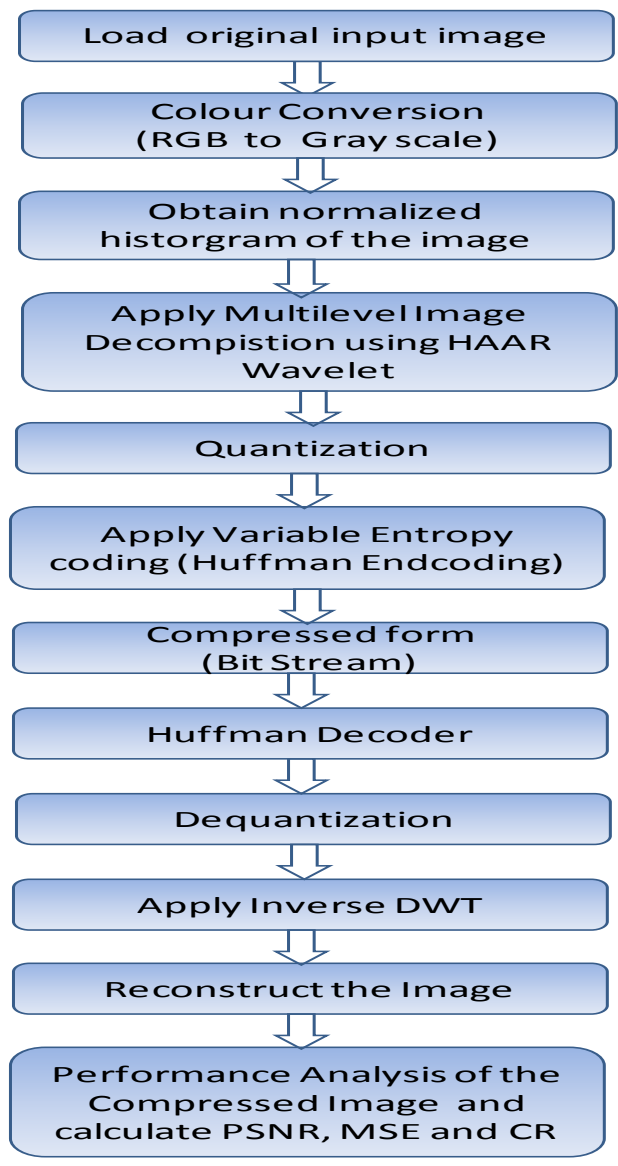

Figure 3 Flow Diagram of the proposed Method
The following figures give the visual and quantitative results of the method considering the hybrid transformation for block size $32 \times 32$. The test medical image (X-ray hand.jpg) is compressed and decompressed using the proposed method.

The following figures depict the output of the algorithm in the sequence of execution.

Figure 4 shows the original image and its gray scale converted form (RGB to $\mathrm{YCbCr}$ transformation for the block size of $32 \times 32$ ). The histogram of the image is used as reference to evaluate the mean squared error of the image.

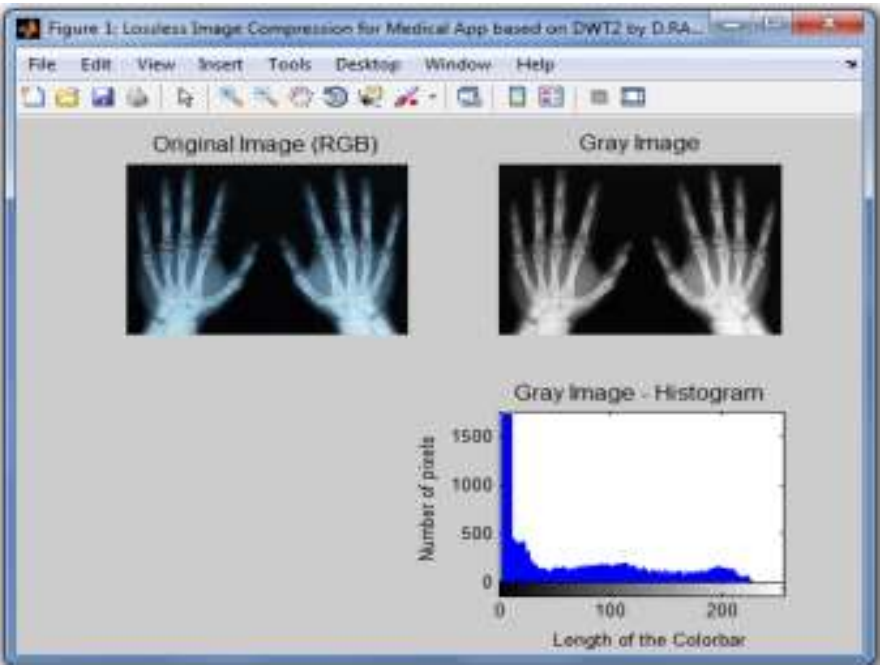

Figure 4 Original Image and Histogram

Figure 5 depicts the decomposed image after applying forward Discrete Wavelet Transform in the First level and second level of DWT2.

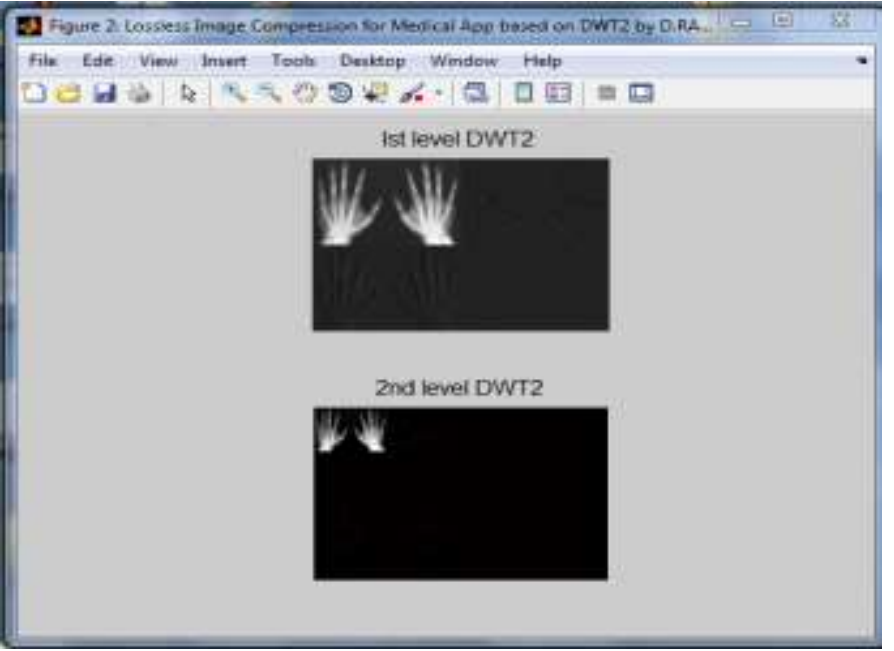

Figure 5 Decomposed Image after applying FDWT 
Figure 6 shows the reconstructed image after applying dequantization, Huffman decoder and Inverse Discrete Wavelet Transform (IDWT2). The mean squared error of the image between original and the reconstructed image along with the histogram is displayed in order to find out the visual and quantitative performance of the proposed compression algorithm.

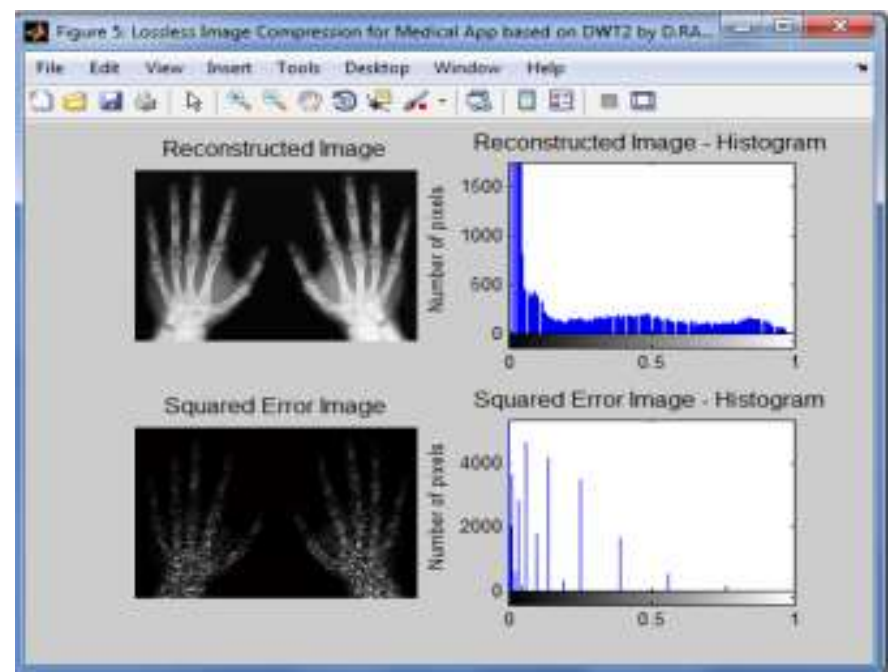

Figure 6 Reconstructed image and its Histogram

\section{Results and Discussions}

The proposed image coding scheme is implemented according to the description in section-III and tested with different image formats of bench mark medical images. A set of test medical images is shown in the Fig 7. The effectiveness of the proposed method is elucidated by means of the experimental results. The proposed method is implemented in MATLAB2014a. The input images used in the experiments include Xray hand.jpg, CT brain.gif, MRI head.jpg, US liver.jpg, X-ray chest.jpg, and CT brain.png format.
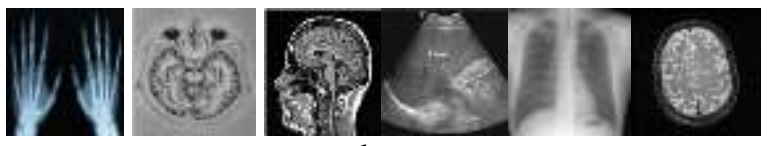

Xray_hand. CT_Brain. MRI_head US_Liver Xray_Chest CT_Brain(png)

Figure 7 Bench mark test medical images

Here compression ratio is measured in terms of Bits Per Pixel (bpp) and image quality in terms of PSNR and visual fidelity index [2]. Performance parameters of the images are determined by measuring the compression ratio, peak signal to noise ratio and mean square error of the compressed images. Table-1 summarizes the experimental performance under different image formats of medical images.
TABLE 1 EXPERIMENTAL PERFORMANCE UNDER DIFFERENT IMAGE FORMATS OF MEDICAL IMAGES

\begin{tabular}{|c|c|c|c|c|c|c|c|}
\hline $\begin{array}{l}\text { Types } \\
\text { of } \\
\text { Images }\end{array}$ & $\begin{array}{l}\text { Image } \\
\text { Format }\end{array}$ & MSR & $\begin{array}{c}\text { PSNR } \\
\text { in } d b\end{array}$ & $\begin{array}{c}\text { Or } \\
\text { igi } \\
\text { nal } \\
\text { siz } \\
\text { e }\end{array}$ & $\begin{array}{c}\text { Com } \\
\text { press } \\
\text { ed } \\
\text { size } \\
\text { (kb) }\end{array}$ & CR & $\begin{array}{c}\text { Space } \\
\text { Saving } \\
(\%)\end{array}$ \\
\hline $\begin{array}{l}\text { X-ray } \\
\text { hand }\end{array}$ & jpg/jpeg & $\begin{array}{c}2.7439 \\
\text { E-27 }\end{array}$ & 313.75 & $\begin{array}{r}16 \\
\text { KB }\end{array}$ & 4 & 4 & 75 \\
\hline $\begin{array}{c}\text { CT } \\
\text { Brain }\end{array}$ & gif & $\begin{array}{c}2.8012 \\
\text { E-27 }\end{array}$ & 313.66 & $\begin{array}{r}16 \\
\text { KB } \\
\end{array}$ & 4 & 4 & 75 \\
\hline $\begin{array}{l}\text { MRI } \\
\text { head }\end{array}$ & jpg/jpeg & $\begin{array}{c}3.5310 \\
\text { E-27 }\end{array}$ & 312.65 & $\begin{array}{r}12 \\
\mathrm{~KB}\end{array}$ & 4 & 3 & 66.7 \\
\hline $\begin{array}{c}\text { US- } \\
\text { Liver }\end{array}$ & jpg/jpeg & $\begin{array}{c}3.5130 \\
\text { E-27 }\end{array}$ & 312.67 & $\begin{array}{r}12 \\
\mathrm{~KB}\end{array}$ & 4 & 3 & 66.7 \\
\hline $\begin{array}{r}\text { X-ray } \\
\text { chest }\end{array}$ & jpg/jpeg & $\begin{array}{c}7.9083 \\
\text { E-27 }\end{array}$ & 309.15 & $\begin{array}{r}12 \\
\mathrm{~KB} \\
\end{array}$ & 4 & 3 & 66.7 \\
\hline $\begin{array}{c}\text { CT } \\
\text { brain }\end{array}$ & png & $\begin{array}{c}8.7571 \\
\text { E-27 }\end{array}$ & 308.71 & $\begin{array}{c}20 \\
8 \mathrm{~K} \\
\mathrm{~B}\end{array}$ & 4 & 52 & 98 \\
\hline
\end{tabular}

The peak signal to noise ratio (PSNR) is the ratio between a signal's maximum power and the power of the signal's noise. PSNR is most easily defined via the mean squared error $(M S E)$. Given a noise-free $m \times n$ monochrome image $I$ and its noisy approximation $K, M S E$ is defined as:

$$
M S E=\frac{1}{m n} \sum_{i=0}^{m-1} \sum_{j=0}^{n-1}[I(i, j)-K(i, j)]^{2}
$$

The PSNR (in $\mathrm{dB}$ ) is defined as:

$$
\begin{aligned}
\text { PSNR } & =10 \cdot \log _{10}\left(\frac{M A X_{I}^{2}}{M S E}\right) \\
& =20 \cdot \log _{10}\left(\frac{M A X_{I}}{\sqrt{M S E}}\right) \\
& =20 \cdot \log _{10}\left(M A X_{I}\right)-10 \cdot \log _{10}(M S E)
\end{aligned}
$$

Here, $M A X_{I}$ is the maximum possible pixel value of the image. When the pixels are represented using 8 bits per sample, this is 255 . Figure 8 shows the comparison of PSNR versus types of medical image formats. Medical images which are stored in the JPEG formats give better PSNR values than those that are stored in the PNG format.

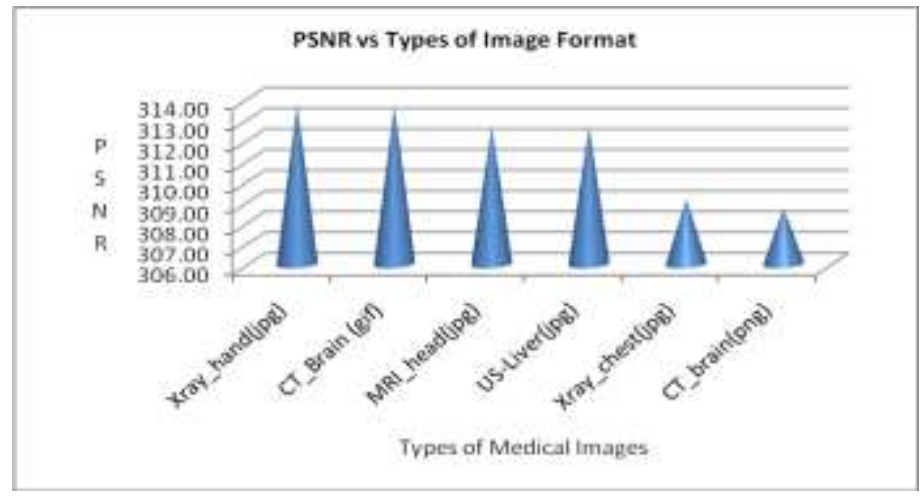

Figure 8 PSNR versus Types of Medical Images 
Compression ratio and Space savings

The compression ratio is the ratio of original image to the compressed image. The space saving of a compression algorithm can be calculated in the following formula.

$$
\text { Space Saving }=\left[1-\frac{\text { Compressed Size }}{\text { Uncompressed Size }}\right]
$$

Figure 9 shows the comparison of space savings versus different types of medical image formats. The proposed algorithm shows that about $57.8 \%$ of space can be saved effectively without losing the quality of the image.

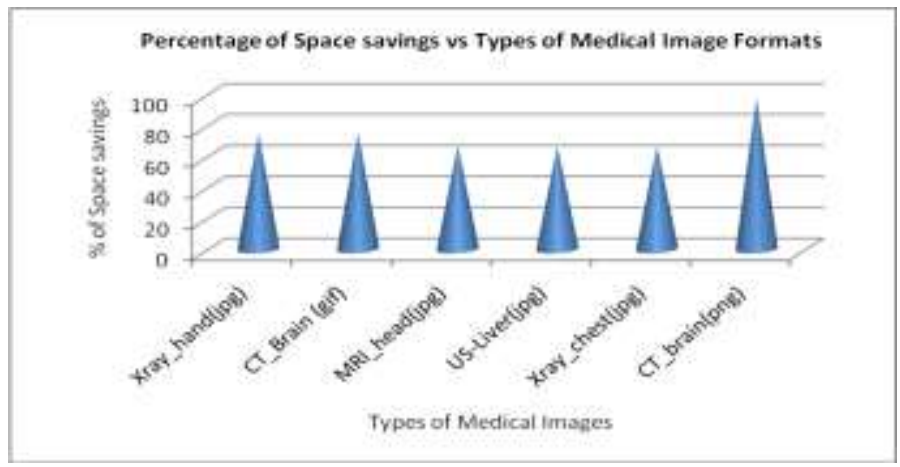

Figure 9 Percentage of space savings versus Types of Image Formats

\section{v. Conclusions And Future Work}

In this proposed paper, we are using hybrid algorithmic approach for medical image compression based on multilevel decomposition using Haar wavelet transform and Huffman variable entropy coding and the image reconstruction is done with wavelet packet. The experimental results show that the proposed hybrid algorithmic approach provides high compression ratio, least mean square error (MSE) and better Peak Signal to Noise ratio (PSNR) between original and reconstructed image. There are various possible directions for future investigations using evolutionary computation such as Fuzzy Logic, Genetic Algorithms (GA) and Artificial Neural Networks (ANN) in order to optimize compression techniques.

\section{Acknowledgment}

The authors would like to express their gratitude to the management of Aurora group of colleges, Hyderabad where this work was performed and thank Dr Suresh Babu, Professor in CSE, for helpful discussions during the course of this work.

\section{References}

[1] Proc. IEEE (Special Issue on Wavelets), vol. 84, Apr. 1996.

[2] S. Grgic, M. Grgic, and B. Zovko-Cihlar,"Performance Analysis of Image Compression Using Wavelets," IEEE Trans. Industrial Electronics, vol. 48, no. 3, pp. 682-695, June 2001.

[3] G.C. Kagadis, C. Kloukinas, K.Moore, and W.R. Hendee, "Cloud Computing in Medical Imaging," American Association of Physicists in Medicine, vol. 40, no.7, pp 1-11, July 2013

[4] A. Paul, T.Z. Khan, P. Podder, R.Ahmed, and M.M. Rahman,'Iris Image Compression using Wavelets Transform Coding," IEEE - 2nd Int Conf on Signal Processing and Integrated Networks (SPIN)-15, pp.544-548, 2015.

[5] Z.Y Songyu, Y.G.Zhou and Y. Mao , "Medical Image Compression Based on Wavelet Transform," IEEE- Conf. Proceedings of ICSP '98, pp. 811-814, 1998.

[6] L. Bo, and Y. Zhaorong, "Image Compression Based on Wavelet Transform," IEEE-Int Conf on Measurement, Information and Control (MIC), pp.145-148, May 18-20, 2012.

[7] J. Zukoski,T. Boult, and T.Lyriboz, "A Novel Approach to Medical Image Compression," Int. J of Bioinformatics Res and App, vol.2, no.1, pp. 89-103, 2006

[8] C.Doukas and I.Maglogiannis, "Region of Interest Coding Techniques for Medical Image Compression" "' IEEE Engineering In Medicine And Biology Magazine, pp. 29-35, Sep/Oct. 2007.

[9] M. Antonini, M. Barland, P. Mathieu, and I. Daubechies, "Image coding using the wavelettransform," IEEE Trans. Image Processing, vol. 1, pp. 205-220, Apr. 1992.

[10] K. K. Simhadri, S. S. Iyengar, R.J.Holyer, M. Lybanon, and J.M. Zachary, Jr., "Wavelet-Based Feature Extraction from Oceanographic Images ," IEEE Trans. Geoscience and Remote Sensing, vol. 36, no. 3, pp. 767-778, May 1998.

[11] W.Yannan, Z. Shudong, and L. Hui, "Study of Image Compression Based on Wavelet Transform," Proc. IEEE- Fourth Int Conf on Intelligent Systems Designand Engineering Applications, pp. 575578, 2013.

[12] S Mallat, "A theory of multiresolution signal decomposition: The wavelet representation," IEEE Trans. Pattern Anal. Machine Intell.,vol. 11, pp. 674-693, July 1989.

[13] S Mallat, "Multifrequency channel decomposition of images and wavelet models," IEEE Trans. Acoust., Speech, Signal Processing, vol.37, pp. 2091-2110, Dec. 1989

[14] B. E. Usevitch, "A Tutorial on Modern Lossy Wavelet Image Compression Foundations of JPEG2000," IEEE Signal Processing Magazine, pp. 22-35,Sep. 2001.

[15] M. Smith and S. Eddins, "Analysis/synthesis techniques for subband image coding," IEEE Trans.Acoust. Speech Signal Processing, vol. 38, pp. 1446-1456, Aug. 1990. 